\title{
MODELING OF MECHANISMS OF QUASI-CONSTANT MOVEMENT
}

\author{
Imants Nulle, Eriks Kronbergs, Aivars Kakitis, Olafs Vronskis \\ Latvia University of Life Sciences and Technologies, Latvia \\ imants.nulle@1lu.lv, eriks.kronbergs@1lu.lv, aivars.kakitis@1lu.lv, olafs.vronskis@1lu.lv
}

\begin{abstract}
In practice for material pressing mechanisms are used that provide synchronous motion of components. Gears can satisfy this design specification accurately, but they are more expensive and difficult to manufacture than linkages, and not convenient to transmit the motion between two parallel axis with large center distance. Thus, when a quasi-constant transmission ratio within a wide range of driving link rotation is allowed, four-bar linkages are suitable to replace gears. The possibility of using a four-bar linkage mechanism to ensure opposite rotation of double cranks is analysed. A planar four-bar linkage is characterized by having four revolute joints with parallel axis. The synthesis of four-bar linkages will yield an approximation of transmission ratio between the input and output crank rotation. Crank displacement equations for antiparallelogram and similar forbar linkage mechanisms depending on the sizes of the links are presented. Antiparallelogram mechanism with ratios $j=30$ between the center-distance and the crank length provide a transmission ratio $u_{13}=1 \pm 5 \%$ for driving the crank angle in range 40-140 deg. The Matlab simulation showed quasi-constant transmission ratio $u=1 \pm 2 \%$ for the four-bar mechanisms with coupler length not equal with fixed center distance.
\end{abstract}

Keywords: four-bar linkage, quasi-constant motion, kinematic parameters.

\section{Introduction}

Motions with uniform transmission ratio in a determined range are required for wide range of mechanisms. For example, material pressing mechanisms and wood feeding equipment [5] can be mentioned. In log feeding plants different double crank mechanisms are used. Their main task is the alignment of logs with different and irregular shapes [9]. For agricultural sprayers simultaneous opposite rotation of right and left sprayer booms is necessary for preparation of transport or working condition.

Gears and cams can satisfy these design tasks accurately, but they are more expensive and difficult to manufacture than linkages. Gear mechanisms are not suitable to transmit the motion between two parallel axis with large center distance. Thus, when a quasi-constant transmission ratio of double crank opposite rotation is necessary, four-bar linkages can replace circular gears or cams [5].

The parallelogram mechanism has double cranks, but it transmits a uniform angular velocity ratio of the cranks on the same direction. If the links of parallelogram linkages are in a crossed position the result is the so-called antiparallelogram linkage. Although a complete revolution of both cranks is possible, they do not move in unison; they rotate in opposite directions, and in the dead-center position they might cross back to a parallelogram [7]. Finding out the relationship between the double crank turning angles and the possibility of ensuring a constant transmission ratio is the purpose of this work. The angle of the driving crank in this study is limited to $<180 \mathrm{deg}$, taking into account the peculiarities of the uncertainty of the mechanism state. At the same time with the antiparalelogram kinematics study, a similar four-bar mechanism analysis has been performed. Similar four-bar mechanism differs from antiparallelogram with different coupler lengths that differ from the distance between the fixed joints. This type of linkages can ensure quasi-constant transmission ratio relationship between motion parameters of double cranks with least deviation. To achieve this goal, mathematical modelling [2, 3, 6, 8, 10,11] was performed using MathCad and MatLab software.

\section{Materials and methods}

The ratio of the anti-paralelogram mechanism of two cranks $\mathrm{AB}$ and $\mathrm{CD}$ in accordance with the parameters in Fig. 1 is given [1] by the equations (1).

$$
u_{13}=\frac{E D}{A E}
$$

This relation must be expressed in terms of the angular rotation of the cranks. Knowing that:

$$
\triangle A B E=\triangle C D E
$$

follows 


$$
E D \cdot h_{2}=A E \cdot h_{1}
$$

then

$$
\frac{E D}{A E}=\frac{h_{1}}{h_{2}}=\frac{\sin \varphi}{\sin \psi} \Rightarrow u_{13}=\frac{E D}{A E}=\frac{\sin \varphi}{\sin \psi}
$$

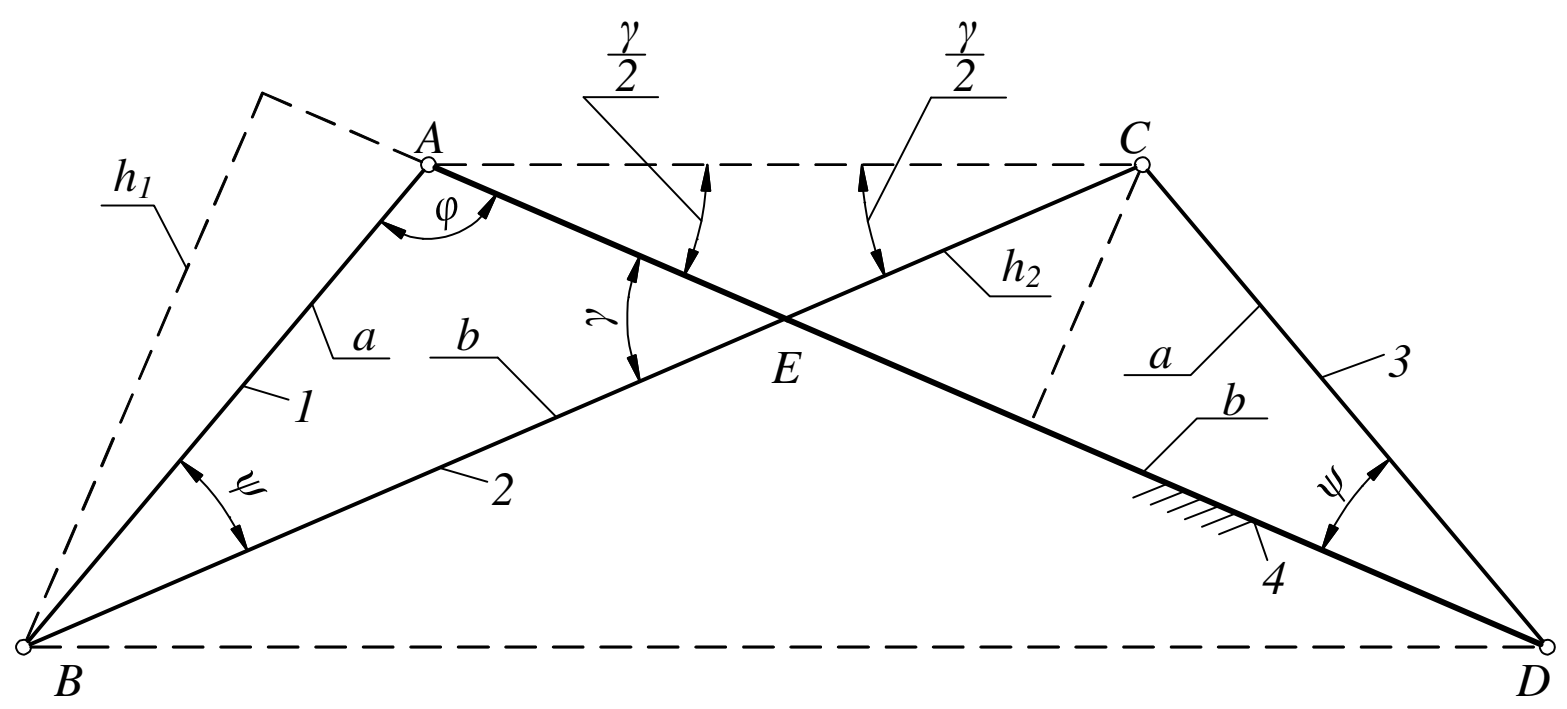

Fig .1. Parameters of antiparallelogram mechanism

The relationship between the double crank turning angles is as follows:

$$
\varphi=\pi-(\gamma+\psi) \text {. }
$$

The angle $\gamma \mathrm{g}$ can be expressed:

$$
\gamma=2 \arcsin \left(\frac{a \cdot \sin \psi}{\sqrt{b^{2}+a^{2}-2 a b \cdot \cos \psi}}\right),
$$

Then:

$$
\varphi=\pi-\left(2 \arcsin \left(\frac{a \cdot \sin \psi}{\sqrt{b^{2}+a^{2}-2 a b \cdot \cos \psi}}\right)+\psi\right)
$$

Equations (4) and (7) are used to simulate the antiparallelogram mechanism in the MathCad software to determine the relationship between the rotation angles of the cranks $\varphi$ and $\psi$ depending on the size of the cranks and the coupler.

Similar four-bar mechanism differs from antiparallelogram with different coupler lengths that differ from the distance between the fixed joints. When designing equipment, the parameters such as: fixed joint distance, required rotation angle and approximate cranks length are given. The angular location of the cranks depending on the length of the coupler size is necessary to be determined. The main idea of this method is that the mechanism, which cranks rotate with a constant transmission ratio $u=1$, must maintain a quasi-constant length of the coupler with least deviation.

The length of the coupler $l$ is expressed by trigonometric functions (Fig. 2), such as the length of the line in the $\mathrm{x}-\mathrm{y}$ coordinates. For calculations MS Excel software was used. The length of the coupler is calculated in dependence of the angles of the cranks $\varphi_{1}$ and $\varphi_{2}$. The displacement equation of the four-bar linkage may be obtained considering a rectangular coordinate system, with respect to which the coordinates of revolute joints may be written as a functions of the revolution angle.

Let us accept the following terms: driving cranklength $-a_{1}$, driven cranklength $-a_{2}$, distance between fixed revolute joints $-b$ and coupler length $-l$ (Fig. 2). 
Assuming that $l \neq b$, at the driving link position $\varphi_{1}=0$, the driven link position will not be equal to zero, let us assume it $\varphi_{0}$. To describe the kinematics of this mechanism, according to Fig. 2 the following geometric equations were established:

$$
l^{2}=\left(x_{2}-x_{1}\right)^{2}+\left(y_{1}+y_{2}\right)^{2} .
$$

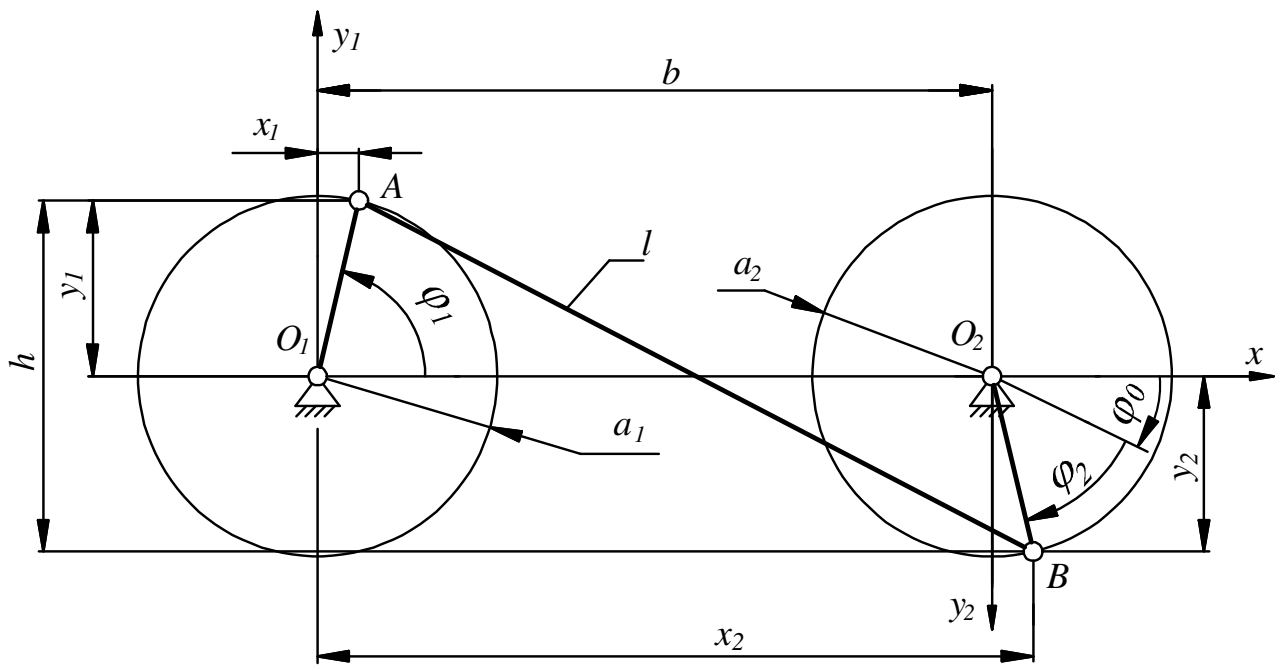

Fig. 2. Mechanism scheme for creating kinematic model

$$
\begin{gathered}
x_{1}=a_{1} \cdot \cos \varphi_{1} \text { and } x_{2}=b+a_{2} \cdot \cos \left(\varphi_{2}+\varphi_{0}\right) \\
y_{1}=a_{1} \cdot \sin \varphi_{1} \text { and } y_{2}=a_{2} \cdot \sin \left(\varphi_{2}+\varphi_{0}\right)
\end{gathered}
$$

Inserting formulas (9) and (10) in equation (8), was obtained:

$$
l^{2}=\left(b+a_{2} \cdot \cos \left(\varphi_{2}+\varphi_{0}\right)-a_{1} \cdot \cos \varphi_{1}\right)^{2}+\left(a_{1} \cdot \sin \varphi_{1}+a_{2} \cdot \sin \left(\varphi_{2}+\varphi_{0}\right)\right)^{2} .
$$

Making simplifications, we obtain an expression for calculating the length of the coupler according to the angles of rotation of the cranks (12):

$$
l=\sqrt{b^{2}+a_{1}^{2}+a_{2}^{2}+2 b \cdot\left(a_{2} \cdot \cos \left(\varphi_{2}+\varphi_{0}\right)-a_{1} \cdot \cos \varphi_{1}\right)-2 a_{1} \cdot a_{2} \cos \left(\varphi_{1}+\varphi_{2}+\varphi_{0}\right)} .
$$

Let us assume that the rotation angles are the same, $\varphi_{1}=\varphi_{2}$. and determine the required length of the coupler $l$. To determine the impact of the crank length on the mechanism rotation equability, we denote the ratio of the lengths of the cranks $k=a_{2} / a_{1}$.Substituting $a_{2}$ in equation (12) with $a_{2}=k \cdot a_{1}$ and $\varphi_{1}=\varphi_{2}$, the length of the coupler $l$ can be calculated according to equation (13):

$$
l=\sqrt{b^{2}+a_{1}^{2}\left(1+k^{2}\right)+2 b \cdot a_{1} \cdot\left(k \cdot \cos \left(\varphi_{1}+\varphi_{0}\right)-\cos \varphi_{1}\right)-2 a_{1}^{2} \cdot k \cdot \cos \left(2 \varphi_{1}+\varphi_{0}\right)} .
$$

To determine in more detail the link sizes of the mechanism for further mathematical modelling previous equation (13) calculations were made for $b=1000 \mathrm{~mm}$ and $a_{1}=50 \mathrm{~mm}$ (Fig. 3).

From Fig. 3 we can conclude that by changing the initial angle $\varphi_{0}$ of the driven crank (that determines the length of the coupler), we can obtain an area, in which the relation between the angles $\varphi_{1}$ and $\varphi_{2}$ is quasi-constant (turning angle between 40 to 130 degrees).

In order to find out the effect of the crank length ratio $k$ on smooth motion, a simulation of the motion of the mechanism was performed with different values of the ratio $k$ (Fig. 4).

The obtained results prove that the crank inequality increases the asymmetry of the mechanism's operation and, consequently, unevenness of the rotation. The results of simulation prove that the best result can be obtained with the ratio $k=1$ (Fig. 4).

Further simulations are made using equal cranks lengths $a_{1}=a_{2}=a$. Considering that the transmission ratio is expressed as the ratio of angular velocities, a Matlab-Simulink model was created that allows to detect both the change in the rotation angle and angular velocity of the driven crank. 
Matlab-Simulink diagram was created according to equation (14) derived from equation (11). Initial angle of the driven crank was assumed equal zero.

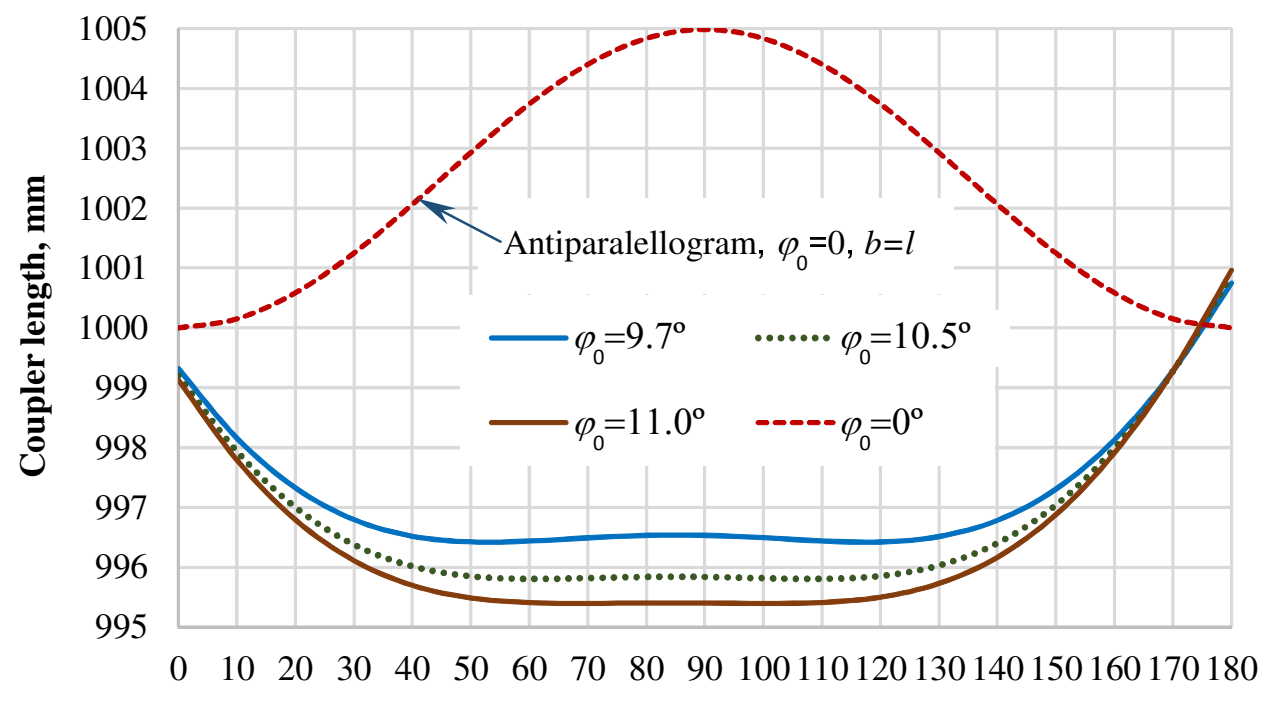

Driving crank angle $\varphi_{1}$, deg

Fig. 3. Changes of coupler length depending on driving angle for different initial angle $\varphi_{0}, k=1$

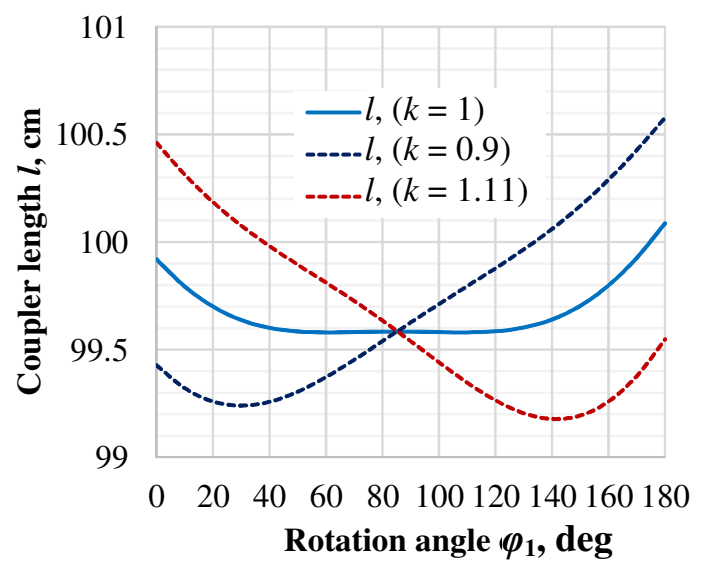

Fig. 4. Impact of crank ratio $k$ on smoothness of mechanism motion

The driven crank angle depending on the driving crank angle was calculated by the formula:

$$
\varphi_{2}=\arccos \left(\cos \varphi_{1}+\frac{l^{2}-b^{2}}{2 b \cdot a}-\frac{a}{b}\left(1-\cos \left(\varphi_{1}+\varphi_{2}\right)\right)\right) .
$$

Angular velocity of the driven crank was calculated in Matlab-Simulink by differentiation of the rotation angle with respect to the time. The transmission ratio $u$ was also determined between the driving $\omega_{1}$ and driven crank $\omega_{2}$ angular velocities by formula (15):

$$
u=\frac{\omega_{1}}{\omega_{2}} .
$$

\section{Results and discussion}

A model for the antiparallelogram mechanism was created and simulated using the Mathcad software. As a result, curves have been obtained, which show the dependence of the angles of the driving and driven cranks (Fig. 5) for different ratios $j$ between the center-distance $b$ and the crank length $a$. Ratio $j$ was calculated according to equation (16): 


$$
j=\frac{b}{a} .
$$

The graphs in Fig. 5 show that as the ratio $j$ increases, the rotational relationship between the driving and driven angles becomes more linear.

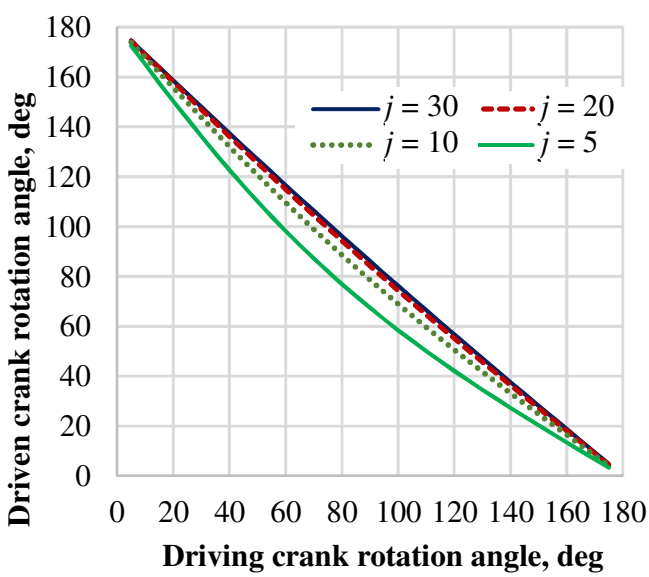

Fig. 5. Relationship between angles of driving and driven cranks

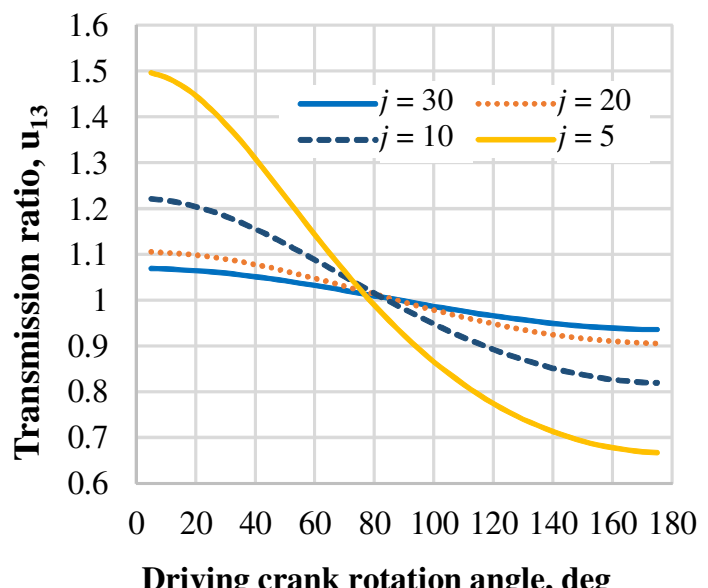

Fig. 6. Transmission ratio depending on angle of driving crank for antiparalellogram

The graphs in Fig. 6 show that for ratios $j=30$ between the center-distance $b$ and the crank length $a$ the transmission ratio $u_{13}=1 \pm 5 \%$ for the driving crank angle is in range 40-140 deg.

The results obtained by simulating the Matlab-Simulink model are shown in Figure 7.

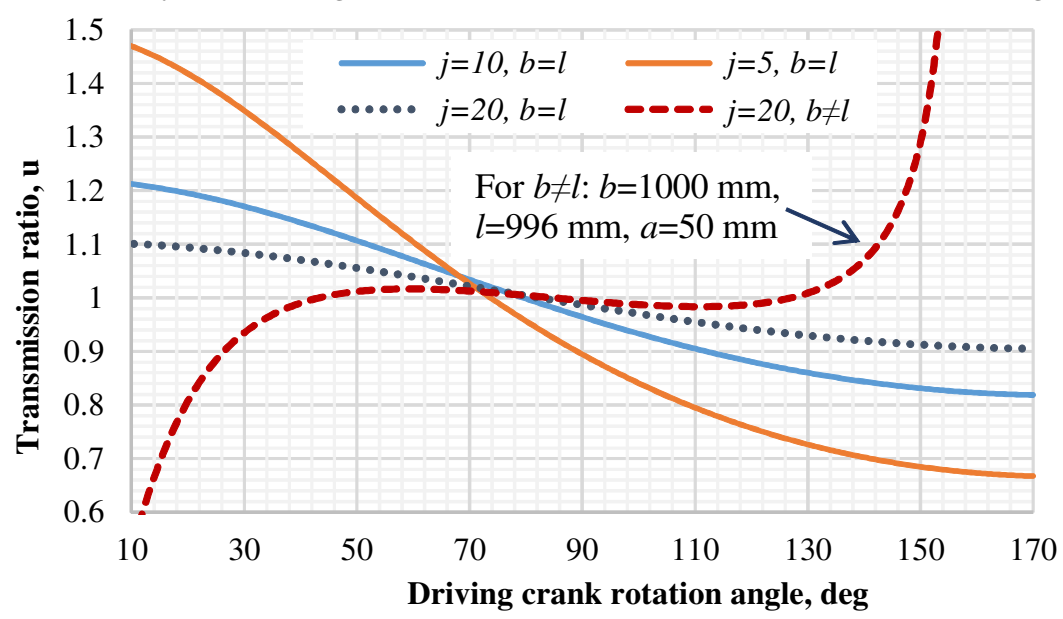

Fig. 7. Transmission ratio depending on driving crank angle

It can be seen that the transmission ratios for the antiparallelogram correspond to the results obtained in the Mathcad model (curves with $b=l$ ). The red-dashed line shows a change in the transmission ratio if the distance between the centres $b$ is not equal to the coupler length $l$. The simulation showed that the transmission ratio is quasi-constant for the coupler length $l=996 \mathrm{~mm}$ between the driving crank turning angles in range 40 to 130 degrees. In this range, the transmission ratio $u=1 \pm 2 \%(j=20)$. It is 2.5 times less than for the antiparallelogram with the ratio $j=30$.

During the simulation of the mechanism with Matlab-Simulink, a change in the angles of the driving and driven cranks was determined (Fig. 8). Assuming that the initial angle of the driving crank $\varphi_{1}=0$, the driven crank angle $\varphi_{2}=24$ degrees. The linear relationship between the rotational angles of the two cranks begins at $\varphi_{1}=40$ degrees. The linear phase ends, when the rotational angle of the driving crank reaches 130 degrees. This situation provides a good torque transmission for the mechanism.

It was found, for example, that the material pressing mechanism, mentioned before a quasiconstant transmission rate, can be provided for fixed center distance $b=400 \mathrm{~mm}$ and the cranks length 
of $60 \mathrm{~mm}$. This means that the ratio of the fixed center distance to the cranks length is $j=6.67$. It was found that in this case the best length of the coupling is $l=387 \mathrm{~mm}$ (Fig. 9).As shown in Fig. 9, the maximum deviation in the quasi-constant phase depends on the length of the coupler.

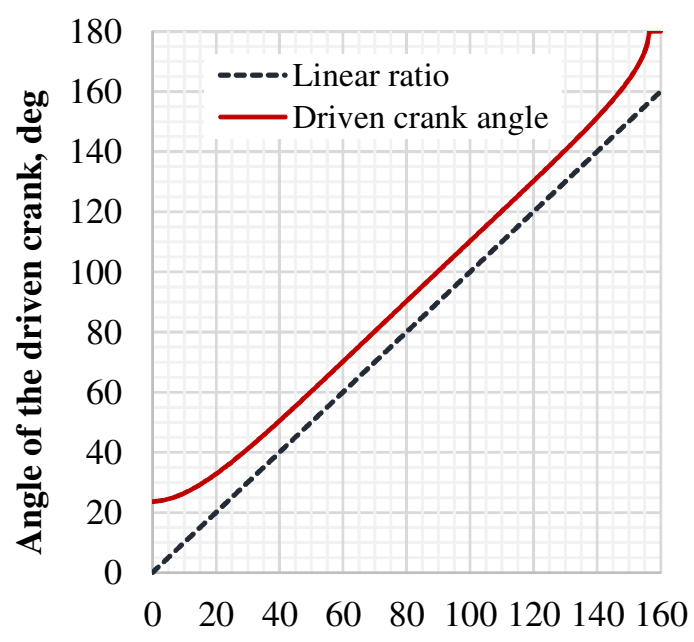

Angle of the driving crank, deg

Fig. 8. Driven crank rotation angle depending on driving crank rotation angle, $j=20, b=1000 \mathrm{~mm}, l=996 \mathrm{~mm}, a=50 \mathrm{~mm}$

Assuming that the gear ratio can vary in the range of $\pm 2 \%$, we can determine the permissible turning range. For a coupler with length $l=387 \mathrm{~mm}$, it lays from 40 to 110 degrees.

The reduction of the length of the coupler to $l=386 \mathrm{~mm}$ reduces the change in the transmission ratios to $\pm 1.5 \%$, but also reduces the range of the turning angles.

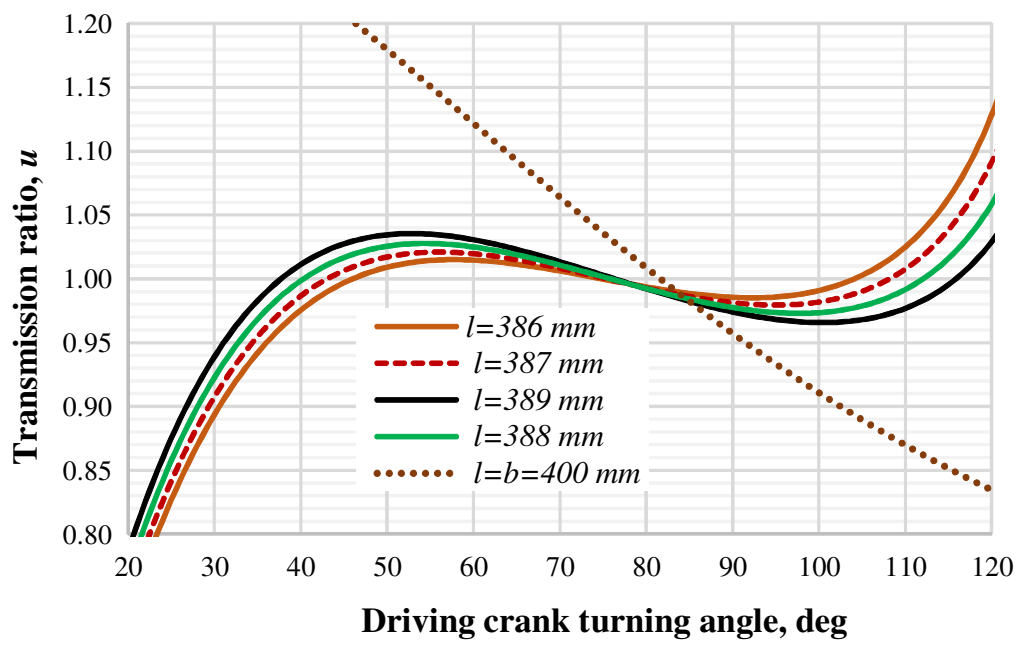

Fig. 9Transmission ratio depending on driving crank angle $b=400 \mathrm{~mm}, a=60 \mathrm{~mm}$

Figure 9 shows the change in the transmission ratio also for the anti-parallelogram $(l=b)$ with $j=6.77$. It can be seen that it is not possible to get a quasi-constant phase of acceptable length. For example, for the material pressing mechanism it is possible to obtain quasi-constant transmission ratio $u=1 \pm 2 \%$ in the range of $40-110$ deg of the driving crank angle.

\section{Conclusions}

1. Antiparallelogram mechanism with ratios $j=30$ between the center-distance $\mathrm{b}$ and the crank length provides a transmission ratio $u_{13}=1 \pm 5 \%$ for the driving crank angle in the range 40$140 \mathrm{deg}$. As the ratio $j$ increases, the rotational relationship between the driving and driven angles becomes more linear. 
2. The Matlab simulation showed that the transmission ratio is quasi-constant for the coupler length $l=996 \mathrm{~mm}(b=1000 \mathrm{~mm})$ between the driving crank turning angles in the range 40 to 130 degrees. In this range, the transmission ratio $u=1 \pm 2 \%(j=20)$. It is 2.5 times less than for the antiparallelogram with the ratio $j=30$.

3. For example, for the material pressing mechanism (for fixed center distance $b=400 \mathrm{~mm}$ and the cranks length of $60 \mathrm{~mm}$ ) it is possible to obtain quasi-constant transmission ratio $u=1 \pm 2 \%$ in the range of 40-110 deg of the driving crank angle, if the coupler length is $387 \mathrm{~mm}$.

\section{References}

[1] Artobolevsky I. Mechanisms in modern Engineering design.Vol. 1: Lever Mechanisms. Moscow: Mir Publishers, 1979. 632 p.

[2] Dijksmann E.A. Motion Geometry of Mechanisms Cambridge. London: Cambridge University Press, 1976. 288 p.

[3] Erdman A.G., Sandor G.N., Mechanism Design, Analysis and Synthesis. 2nd Edition. New Jersey: Prentice Hall, 1991. 631 p.

[4] Figliolini G., Pennestri E. Synthesis of Quasi-Constant Transmission Ratio Planar Linkages. Journal of Mechanical Design, vol. 137, issue 10, August 19, 2015. 12 p. [online] [10.04.2018]. Available at: $\mathrm{http} / / /$ mechanicaldesign.asmedigitalcollection .asme.org/article. aspx $?$ articleid $=2398101$

[5] Hall A.S. Kinematics and Linkage Design. Prentice-Hall: Englewood Cliffs N. J. 1961 162.p

[6] Hunt K.H. Kinematic Geometry of Mechanisms. New York: Oxford University Press, 1990. 480 p.

[7] Jensen P.W. Classical and Modern Mechanisms for Engineers and Inventors. New York: Marcel Dekker, 1991. 616 p.

[8] McCarthy M.J., Soh G.S. Geometric Design of Linkages. 2nd Edition. New York: Springer-Verlag, 2011. 448 p.

[9] Pelletier G., Bouchard G.J., Trudel L., Constantineau S. "Compact small diameter log sawmill" US Patent Nr. 5,915,429. Jun 29, 1999.

[10] Vujic D., Radojkovic S. Synthesis procedure of planar bar linkages in infinitesimally close positions. Scientific Journal "Series Mechanical Engineering”, vol.1, No 7, 2000, pp. 835 - 848.

[11] Waldron K.J., Kinzel G.L. Kinematics, Dynamics and Design of Machinery. New York: Wiley, 1999. $640 \mathrm{p}$. 